\title{
Maps that preserve left (right) $K$-Cauchy sequences
}

\author{
Olivier Olela Otafudu (1D \\ School of Mathematical and Statistical Sciences North-West University, Potchefstroom Campus, \\ Potchefstroom 2520, South Africa
}

\begin{abstract}
It is well-known that on quasi-pseudometric space $(X, q)$, every $q^{s}$-Cauchy sequence is left (or right) $K$-Cauchy sequence but the converse does not hold in general. In this article, we study a class of maps that preserve left (right) $K$-Cauchy sequences that we call left (right) $K$-Cauchy sequentially-regular maps. Moreover, we characterize totally bounded sets on a quasi-pseudometric space in terms of maps that preserve left $K$-Cauchy and right $K$-Cauchy sequences and uniformly locally semi-Lipschitz maps.
\end{abstract}

Mathematics Subject Classification (2020). 54E35, 54E50, 54E40, 46A17, 26A16

Keywords. Cauchy sequential regularity, left $K$-Cauchy, bornology, uniform continuity, total boundedness

\section{Introduction}

Let $f:(X, d) \rightarrow\left(Y, d^{\prime}\right)$ be a map between two metric spaces $(X, d)$ and $\left(Y, d^{\prime}\right)$. Then $f$ is called Cauchy sequentially-regular (or Cauchy continuous) if $\left(f\left(x_{n}\right)\right)$ is a Cauchy sequence on $\left(Y, d^{\prime}\right)$ whenever $\left(x_{n}\right)$ is a Cauchy sequence on $(X, d)$. In [12], Snipes investigated the class of Cauchy sequentially-regular maps. He proved that a map $f:(X, d) \rightarrow$ $\left(Y, d^{\prime}\right)$ is uniformly continuous if and only if $f$ preserves parallel sequences. Moreover, He characterized Cauchy sequentially-regular maps in terms of maps that preserve equivalent sequences.

In addition, Snipes observed that the class of Cauchy sequentially-regular maps from the metric space $(X, d)$ into the metric space $\left(Y, d^{\prime}\right)$ sits between the class of uniformly continuous maps from the metric space $(X, d)$ into the metric space $\left(Y, d^{\prime}\right)$ and the class of continous maps from the metric space $(X, d)$ into the metric space $\left(Y, d^{\prime}\right)$. He also proved that a map $f:(X, d) \rightarrow\left(Y, d^{\prime}\right)$ is Cauchy sequentially regular whenever $f:(X, d) \rightarrow\left(Y, d^{\prime}\right)$ is continuous and $(X, d)$ is complete. Finally Snipes in [12] investigated extension maps of Cauchy sequentially-regular maps into a complete metric space.

After Snipes paper [12], the concept of Cauchy sequential regularity got an interest in the community of mathematicians. For instance in [8], Jain and Kundu proved that each Cauchy sequentailly-regular map is uniformly continuous if and only if the completion of a

Email address: olivier.olelaotafudu@nwu.ac.za

Received: 23.10.2020; Accepted: 12.05.2021 
metric space $(X, d)$ is a UC-space ( space on which each continuous function is uniformly continuous).

Furthermore, in [6], Di Maio et al. proved that a metric space is complete if and only if each continuous function defined on it is a Cauchy sequentially-regular map.

Moreover, in [2,3], Beer, characterized Cauchy sequentially-regular maps in terms of a family on which they must be strongly uniformly continuous on totally bounded sets and uniformly continuous on totally bounded sets. Recently, Beer proved that on a metric space $(X, d)$, a subset $B$ of $X$ is totally bounded if and only its image $f(B)$ is $d^{\prime}$-bounded whenever $f:(X, d) \rightarrow\left(Y, d^{\prime}\right)$ is Cauchy sequentially-regular map and $\left(Y, d^{\prime}\right)$ is a metric space. In addition, he showed that a subset $B$ of a metric space $(X, d)$ is totally bounded if and only if its image $f(B)$ is bounded subset of $\mathbb{R}$ whenever $f:(X, d) \rightarrow(\mathbb{R},||$.$) is$ uniformly locally Lipschitz.

It is no doubt that uniform continuity and continuity plays an important role in the study of Cauchy sequentially-regular maps on metric spaces. Observe that for any two quasi-metric spaces $(X, q)$ and $(Y, p)$. If a map $f:(X, q) \rightarrow(Y, p)$ is quasi-unformly continuous (or uniformly continuous), then $f:\left(X, q^{s}\right) \rightarrow\left(Y, p^{s}\right)$ is also uniformly continuous but the converse is not true, in general (see Example 3.3). It is well-know that on a quasipseudometric space, if a sequence is $q^{s}$-Cauchy, then it is left (right) $K$-Cauchy sequence but the converse is not true (see for instance $[4,13]$ ). As might be expected these have led to the conjecture that the maps from a quasi-pseudometric space into another quasipseudometric space that preserve left (right) $K$-Cauchy sequences that we call left (right) $K$-Cauchy sequentially regular maps (see Definition 4.8) need to be studied carefully.

The aim of this paper is a careful study of the above-mentioned conjecture. Moreover, for map $f:(X, q) \rightarrow(Y, p)$, where $(X, q)$ and $(Y, p)$ are quasi-pseudometric spaces, we study connections between left (right) $K$-Cauchy sequentially regular maps and $q^{s}$-Cauchy sequentially maps. We also show that a continuous map from a left (right) Smyth complete quasi-metric space into a quasi-pseudometric space is left (right) $K$-Cauchy sequentiallyregular. Finally, we characterize totally bounded sets on a quasi-pseudometric space in terms of left and right $K$-Cauchy sequentially-regular maps and uniformly locally semiLipschitz maps which extend an important result due to Beer and Garrido ([1, Theorem $3.2]$ ) in our settings.

\section{Preliminaries}

In this section we summarize some basic results on quasi-pseudometric spaces. For more details about quasi-pseudometric spaces we recommend the following articles $[4,7,9,13]$.

Let $X$ be a set and $q: X \times X \rightarrow[0, \infty)$ be a function. Then $q$ is an quasi-pseudometric on $X$ if

(a) $q(x, x)=0$ for all $x \in X$,

(b) $q(x, y) \leq q(x, z)+q(z, y)$ for all $x, y, z \in X$.

If $q$ is a quasi-pseudometric on $X$, then the pair $(X, q)$ is called an quasi-pseudometric space.

If the function $q$ satisfies the condition

(c) for any $x, y \in X, q(x, y)=0=q(y, x)$ implies $x=y$ instead of condition (a), then $q$ is called a $T_{0}$-quasi-metric on $X$ and the pair $(X, q)$ is called $T_{0}$-quasi-metric space (see for instance [9]).

Furthermore, if $q$ is a quasi-pseudometric on $X$, then the function $q^{t}: X \times X \rightarrow[0, \infty)$ defined by $q^{t}(x, y)=q(y, x)$, for all $x, y \in X$ is also a quasi-pseudometric on $X$ and it is called the conjugate quasi-pseudometric of $q$. 
Note that for any $q$ quasi-pseudometric on $X$, the function $q^{s}$ defined by $q^{s}(x, y):=$ $\max \left\{q(x, y), q^{t}(x, y)\right\}$ for all $x, y \in X$ is a pseudometric on $X$, usually called the symmetrised quasi-pseudometric of $q$.

If $(X, q)$ is a quasi-pseudometric space. Then we associete the topology $\tau(q)$ on $X$ with respect to $q$ where its base is given by the family $\left\{D_{q}(x, \epsilon): x \in X\right.$ and $\left.\epsilon>0\right\}$ with $D_{q}(x, \epsilon)=\{y \in X: q(x, y)<\epsilon\}$.

If $A \subseteq X$ and $\epsilon>0$, then the set $D_{q}(A, \epsilon)$ is defined by

$$
D_{q}(A, \epsilon):=\bigcup_{a \in A} D_{q}(a, \epsilon) .
$$

We say that a subset $A$ of $X$ is $q$-totally bounded provided that $A$ is $q^{s}$-totally bounded, i.e. for any $\epsilon>0$, there exists $F \in \mathcal{F}$ with $F \subseteq A \subseteq D_{q^{s}}(F, \epsilon)$, where $\mathcal{F}$ is the collection of all finite subsets of $X$ (see [14, Definition 5]).

In the sequel we denote by $\mathscr{T} \mathscr{O}_{q}(X)$ the collection of all $q$-totally bounded subsets of $X$.

It is well-known that $\mathscr{T O O}_{q}(X)$ forms a bornology on $X$, i.e.

(a) $\{x\} \in \mathscr{T O B}_{q}(X)$ whenever $x \in X$,

(b) if $A \subseteq B$ with $\left.B \in \mathscr{T O P}_{q}(X)\right)$, then $A \in \mathscr{T O S}_{q}(X)$,

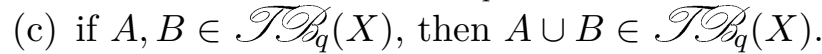

It is easy to see that $\mathscr{T O P}_{q^{s}}(X)={\mathscr{T} \mathscr{B} q^{t}}(X)=\mathscr{T} \mathscr{B}_{q}(X)$.

Definition 2.1. Let $(X, q)$ be a quasi-pseudometric space. A sequence $\left(x_{n}\right)$ in $X$ is called:

(a) left $K$-Cauchy if for any $\epsilon>0$, there exists $N \in \mathbb{N}$ such that

$$
q\left(x_{k}, x_{m}\right)<\epsilon \text { whenever } n, k \in \mathbb{N} \text { with } N \leq k \leq n .
$$

(b) right $K$-Cauchy if for any $\epsilon>0$, there exists $N \in \mathbb{N}$ such that

$$
q^{t}\left(x_{k}, x_{m}\right)<\epsilon \text { whenever } n, k \in \mathbb{N} \text { with } N \leq k \leq n .
$$

(c) $q^{s}$-Cauchy if it is a Cauchy sequence in the symmetrised quasi-pseudometric $q^{s}$.

Note that if we want to emphazise the quasi-pseudometric $q$ on $X$, we shall say that a sequence is right $q$ - $K$-Cauchy and left $q$ - $K$-Cauchy.

For a quasi-pseudometric space $(X, q)$. It well-known that these above three concepts are associated as follows:

$q^{s}$-Cauchy $\Longrightarrow$ left $K$-Cauchy and $q^{s}$-Cauchy $\Longrightarrow$ right $K$-Cauchy.

Remark 2.2. Let $(X, q)$ be quasi-pseudometric space.

(a) A sequence $\left(x_{n}\right)$ in $X$ is left $q$ - $K$-Cauchy if and only if $\left(x_{n}\right)$ is right $q^{t}-K$-Cauchy.

(b) A sequence $\left(x_{n}\right)$ in $X$ is $q^{s}$-Cauchy if and only if the sequence $\left(x_{n}\right)$ is both left $q$ - $K$-Cauchy and right $q$ - $K$-Cauchy.

The following definition can be found for instance on [4].

Definition 2.3. Let $(X, q)$ be quasi-pseudometric space. We say that $(X, q)$ is:

(a) bicomplete if its associated pseudometric space $\left(X, q^{s}\right)$ is complete, that is, every $q^{s}$-Cauchy sequence is $q^{s}$-convergent;

(b) sequentially left (right) $K$-complete if every left (right) $K$-Cauchy sequence is $q$ convergent;

(c) sequentially left (right) Smyth complete if every left (right) K-Cauchy sequence is $q^{s}$-convergent; 


\section{Uniformly continuous and semi-lipschitz maps}

An asymmetric norm on a real vector space $X$ is a function $\| . \mid: X \rightarrow[0, \infty)$ satisfying the conditions

(1) ||$x|=\|-x|=0$ then $x=0$;

(2) $\|a x|=a \| x|$

(3) ||$x+y|\leq||x|+|| y \mid$,

for all $x, y \in X$ and $a \geq 0$. Then the pair $(X, \| \cdot \mid)$ is called an asymmetric normed space.

The conjugate asymmetric norm |.|| of ||.| and the sysmmetrisation norm $\|$.$\| of \| . \mid$ are defined respectively by

$$
|x\|:=\|-x| \text { and } \quad\|x\|:=\max \{|x\|,\| x|\} \text { for any } x \in X .
$$

An asymmetric norm $\| . \mid$ on $X$ induces a quasi-metric $q_{\| . \mid}$on $X$ defined by

$$
q_{\| . \mid}(x, y)=\| x-y \mid \text { for any } x, y \in X \text {. }
$$

If $(X,\|\|$.$) is normed lattice space, then the function \| . \mid$ defined by $\|x \mid:=\| x^{+} \|$, where $x^{+}=\max \{x, 0\}$ is an asymmetric norm on $X$.

A basic but interesting example we point out the asymmetric norm $u$ on $\mathbb{R}$ (considered as a real vector space) defined for any $y \in \mathbb{R}$ by $u(y)=y^{+}$, where $y^{+}=\max \{y, 0\}$, it follows that $u^{t}(y)=\max \{-x, 0\}=y^{-}$and $u^{s}(y)=\max \left\{y^{+}, y^{-}\right\}=|x|$. In addition, the asymmetric norm $u$ induces the quasi-metric $q_{u}$ on $\mathbb{R}$ defined by $q_{u}(x, y)=(x-y)^{+}=\max \{x-y, 0\}$ whenever $x, y \in \mathbb{R}$.

The following is a well-known definition.

Definition 3.1. Let $(X, q)$ and $(Y, p)$ be quasi-pseudometric spaces. A map $f:(X, q) \rightarrow$ $(Y, p)$ is called quasi-uniformly continuous (or uniformly continuous) if for any $\epsilon>0$, there exists $\delta>0$ such that $q(x, y) \leq \delta$, then $p(\varphi(x), \varphi(y))<\epsilon$ for all $x, y \in X$.

Lemma 3.2. Let $(X, q)$ and $(Y, p)$ be quasi-pseudometric spaces. If the map $f:(X, q) \rightarrow$ $(Y, p)$ is uniformly continuous, then the function $f:\left(X, q^{s}\right) \rightarrow\left(Y, p^{s}\right)$ is uniformly continuous.

Example 3.3. We equip $X=\mathbb{R}_{+}=[0, \infty)$ with the quasi-metric $q$ defined by $q(x, y)=$ $(y-x)^{+}$for any $x, y \in[0, \infty)$ and $Y=\mathbb{R}$ is equipped with the $T_{0}$-quasi-metric $p$ defined by $p(x, y)=(y-x)^{+}$for any $x, y \in \mathbb{R}$. Then

(i) the function $f(x)=-\sqrt{x}$ whenever $x \in \mathbb{R}_{+}$is uniformly continuous from $\left(\mathbb{R}_{+},||.\right)$ into $(\mathbb{R},||$.$) .$

(ii) the function $f(x)=-\sqrt{x}$ whenever $x \in \mathbb{R}_{+}$is not uniformly continuous from $\left(\mathbb{R}_{+}, q\right)$ into $(\mathbb{R}, p)$.

Let $(X, q)$ be a quasi-metric space and $(Y, \| . \mid)$ be an asymmetric normed space. Then a map $f:(X, q) \rightarrow(Y, \| \cdot \mid)$ is called semi-Lipschitz if there exists $k \geq 0$ such that

$$
|| f(x)-f(y) \mid \leq k q(x, y) \quad \text { for all } x, y \in X .
$$

The number $k$ satisfying (3.1) is called semi-Lipschitz constant for $f$ and the map $f$ is called $k$-semi-Lipschitz. For more details about semi-Lipschitz maps we recommend the reader to see [5].

Definition 3.4. Let $(X, q)$ be a quasi-metric space and $(Y, \| . \mid)$ be an asymmetric normed space. Then:

(a) A map $f:(X, q) \rightarrow(Y, \| . \mid)$ is called locally semi-Lipschitz provided that for all $x \in X$, then there exists $\delta(x)>0$ such that $\left.f\right|_{D_{q}(x, \delta(x))}$ is semi-Lipschtz. 
(b) A function $f:(X, q) \rightarrow(Y, \| . \mid)$ is called uniformly locally semi-Lipschitz provided that for all $x \in X$, there exists $\delta>0$ ( $\delta$ does not depend to $x$ ) such that $\left.f\right|_{D_{q}(x, \delta)}$ is semi-Lipschtz.

Lemma 3.5. Let $(X, q)$ be a quasi-metric space and $(Y, \| \cdot \mid)$ be an asymmetric normed space. If function $f:(X, q) \rightarrow(Y, \| \cdot \mid)$ is locally semi-Lipschitz, then $f:\left(X, q^{s}\right) \rightarrow(Y,\|\cdot\|)$ is locally semi-Lipschitz.

Proof. Suppose that $f:(X, q) \rightarrow(Y, \| . \mid)$ is locally semi-Lipschitz. Let $x \in X$, there exists $\delta(x)>0$ and $k \geq 0$ such that for any

$$
y, z \in D_{q^{s}}(x, \delta(x)) \subseteq D_{q}(x, \delta(x))
$$

we have

$$
\| f(y)-f(z) \mid \leq k q(y, z) \leq k q^{s}(y, z)
$$

and

$$
\| f(z)-f(y) \mid \leq k q(z, y) \leq k q^{s}(y, z) .
$$

Combining (3.2) and (3.3) for some $k \geq 0$ we have

$$
\|f(y)-f(z)\| \leq k q(y, z) \leq k q^{s}(y, z)
$$

whenever $y, z \in D_{q^{s}}(x, \delta(x))$. Thus the function $f:\left(X, q^{s}\right) \rightarrow(Y,\|\cdot\|)$ is locally semiLipschitz.

Remark 3.6. Let $(X, q)$ be a quasi-metric space and $(Y, \| . \mid)$ be an asymmetric normed space. If a function $f i:(X, q) \rightarrow(Y, \| . \mid)$ is locally semi-Lipschitz, then $\left.\varphi\right|_{D_{q}\left(x, \delta_{x}\right)}$ is continuous whenever $x \in X$ and for some $\delta_{x}>0$.

\section{Left (right) $K$-Cauchy sequentially regular maps}

Definition 4.1 (compare [10, Definition 3.1 and Definition 3.4]). Let $(X, q)$ be a quasipseudometric space. Let $\left(x_{n}\right)$ and $\left(y_{n}\right)$ be sequences in $X$.

(a) We say that the sequences $\left(x_{n}\right)$ and $\left(y_{n}\right)$ are parallel with respect to $q$ (noted by $\left.\left(x_{n}\right) \|_{q}\left(y_{n}\right)\right)$ if for any $\epsilon>0$, there exists $n_{\epsilon} \in \mathbb{N}$ such that $q\left(x_{n}, y_{n}\right)<\epsilon$ whenever $n \geq n_{\epsilon}$.

(b) We say that the sequences $\left(x_{n}\right)$ and $\left(y_{n}\right)$ are equivalent with respect to $q$ (noted by $\left.\left(x_{n}\right) \equiv_{q}\left(y_{n}\right)\right)$ if for any $\epsilon>0$, there exists $n_{\epsilon} \in \mathbb{N}$ such that $q^{s}\left(x_{k}, y_{n}\right)<\epsilon$ whenever $n, k \geq n_{\epsilon}$.

Note that the concept of parallel sequences in quasi-pseudometric spaces is not new. For instance, in [10], Moshoko introduced concepts of parallel sequences and equivalent sequences in order to study extensions of maps that preserve $q^{s}$-Cauchy sequences in a quasi-pseudometric space $(X, q)$. But it is well-known that on a quasi-pseudometric space $(X, q)$, any $q^{s}$-Cauchy sequence in $X$ is left $K$-Cauchy (right $K$-Cauchy), still the converse is not true in general. Our definitions of parallel and equivalent sequences are motivated from metric point of view of parallel and equivalent sequences (see [12]) and by the fact that parallel sequences are preserved by uniformly continuous maps and equivalent sequences are preserved by Cauchy-sequentially-regular maps. However, we are studying maps that preserve left $K$-Cauchy (right $K$-Cauchy) sequences. This explains why our Definition 4.1(2) is more general than [10, Definition 3.4]. We point out that in [7], Doitchinov introduced the concept of cosequence sequences which is similar to the concept of parallel sequences with connections to Cauchy sequences in a quasi-pseudometric space. From cosequence sequences, he defined equivalent sequences for a quasi-metric space satisfying some properties (that he called balanced quasi-metric space).

Lemma 4.2. Let $(X, q)$ be a quasi-pseudometric space. Let $\left(x_{n}\right)$ and $\left(y_{n}\right)$ be sequences in $X$ and $a \in X$. If $\left(x_{n}\right)$ is $q^{s}$-convergent to $a$ and $\left(y_{n}\right)$ is $q^{s}$-convergent to $a$, then $\left(x_{n}\right) \equiv_{q}\left(y_{n}\right)$. 
Proof. Let $\epsilon>0$. Suppose that $\left(x_{n}\right)$ is $q^{s}$-convergent to $a$ and $\left(y_{n}\right)$ is $q^{s}$-convergent to $a$. We show that $\left(x_{n}\right) \equiv_{q^{t}}\left(y_{n}\right)$. Then there exists $n_{\epsilon} \in \mathbb{N}$ and $n_{\epsilon}^{\prime} \in \mathbb{N}$ such that

$$
q^{s}\left(a, x_{n}\right)<\frac{\epsilon}{2} \quad \text { if } \quad n \geq n_{\epsilon}
$$

and

$$
q^{s}\left(y_{n}, a\right)<\frac{\epsilon}{2} \quad \text { if } \quad n \geq n_{\epsilon}^{\prime} .
$$

Let $N=\max \left\{n_{\epsilon}, n_{\epsilon}^{\prime}\right\}$. If $N \leq k, n$, then

$$
\begin{aligned}
q^{s}\left(x_{k}, y_{n}\right) & =q^{s}\left(y_{n}, x_{k}\right) \leq q^{s}\left(y_{n}, a\right)+q^{s}\left(a, x_{k}\right) \\
& <\frac{\epsilon}{2}+\frac{\epsilon}{2} \\
& =\epsilon
\end{aligned}
$$

Hence, $\left(x_{n}\right) \equiv_{q}\left(y_{n}\right)$.

The following lemma is a consequence of the definition and Remark 2.2.

Lemma 4.3. Let $(X, q)$ be a quasi-pseudometric space. Let $\left(x_{n}\right)$ and $\left(y_{n}\right)$ be sequences in $X$. If $\left(x_{n}\right) \equiv_{q}\left(y_{n}\right)$, then the sequence $\left(x_{n}\right)$ is left $K$-Cauchy and right $K$-Cauchy.

We leave the proof of the following lemma.

Lemma 4.4. Let $(X, q)$ be a quasi-pseudometric space and $\left(x_{n}\right)$ and $\left(y_{n}\right)$ be any two sequences in $X$ and $a \in X$. If $\left(x_{n}\right)$ is q-convergent to $a$ and $\left(y_{n}\right) \|_{q}\left(x_{n}\right)$, then $\left(y_{n}\right)$ is $q$-convergent to a.

Lemma 4.5. Let $(X, q)$ be a quasi-pseudometric space and $\left(x_{n}\right)$ and $\left(y_{n}\right)$ be any two sequences in $X$. It is true that $\left(x_{n}\right) \equiv_{q}\left(y_{n}\right)$ if and only if the sequence $\left(z_{n}\right)$ is left $K$ Cauchy and right $K$-Cauchy, where $z_{n}:=\left(x_{1}, y_{1}, x_{2}, y_{2}, x_{3}, y_{3}, \cdots\right)$.

Proof. $(\Rightarrow)$ Let $\epsilon>0$. Suppose that $\left(x_{n}\right) \equiv_{q}\left(y_{n}\right)$. Then there exists $n_{\epsilon} \in \mathbb{N}$ such that

$$
q^{s}\left(x_{k}, y_{m}\right)<\epsilon \quad \text { whenever } k, m \geq n_{\epsilon} .
$$

It follows that the sequence $z_{n}=\left(x_{1}, y_{1}, x_{2}, y_{2}, x_{3}, y_{3}, \cdots\right)$ is $q^{s}$-Cauchy sequence. Hence the sequence $z_{n}$ is left $K$-Cauchy and right $K$-Cauchy.

$(\Leftarrow)$ Suppose that the sequence $z_{n}=\left(x_{1}, y_{1}, x_{2}, y_{2}, x_{3}, y_{3}, \cdots\right)$ is left $K$-Cauchy and right $K$-Cauchy. Then the sequence $z_{n}=\left(x_{1}, y_{1}, x_{2}, y_{2}, x_{3}, y_{3}, \cdots\right)$ is $q^{s}$-Cauchy. Therefore, we have that $\left(x_{n}\right) \equiv_{q}\left(y_{n}\right)$ by [12, Theorem 1 (4)].

The following proposition is obvious. Therefore, we omit the proof.

Proposition 4.6 (compare [10, Theorem 3.2]). Let $(X, q)$ and $(Y, p)$ be quasi-pseudometric spaces. Then the following statements are equivalent.

(1) The map $f:(X, q) \rightarrow(Y, p)$ is uniformly continuous.

(2) Whenever $\left(x_{n}\right) \|_{q}\left(y_{n}\right)$ in $X$ and $f:(X, q) \rightarrow(Y, p)$ is a map, then $\left(f\left(x_{n}\right)\right) \|_{p}\left(f\left(y_{n}\right)\right)$ in $Y$.

Remark 4.7. We point out that it is easy to find an example of two sequences which are parallel with respect to $q$ but they are not parallel with respect to $q^{t}$.

Definition 4.8. Let $(X, q)$ and $(Y, p)$ be quasi-pseudometric spaces. A map $f:(X, q) \rightarrow$ $(Y, p)$ is called:

(a) A left $K$-Cauchy sequentially-regular if for any left $K$-Cauchy sequence $\left(x_{n}\right)$ in $X$, then the sequence $\left(f\left(x_{n}\right)\right)$ is left $K$-Cauchy in $Y$.

(b) A right $K$-Cauchy sequentially-regular if for any right $K$-Cauchy sequence $\left(x_{n}\right)$ in $X$, then the sequence $\left(f\left(x_{n}\right)\right)$ is left $K$-Cauchy in $Y$. 
Proposition 4.9. Let $(X, q)$ and $(Y, p)$ be quasi-pseudometric spaces and $f:(X, q) \rightarrow$ $(Y, p)$ be a map. Then we have that the map $f$ is left $K$-Cauchy sequentially-regular in $X$ if and only if whenever $\left(x_{n}\right) \equiv_{q}\left(y_{n}\right)$ in $X$, then $\left(f\left(x_{n}\right)\right) \equiv_{p}\left(f\left(y_{n}\right)\right)$ in $Y$.

Proof. $(\Rightarrow)$ Suppose that $f$ is left $K$-Cauchy and right $K$-Cauchy sequentially-regular. If $\left(x_{n}\right) \equiv_{q}\left(y_{n}\right)$ in $X$, then it follows that the sequence $\left(x_{1}, y_{1}, x_{2}, y_{2}, \cdots\right)$ is left $K$-Cauchy and right $K$-Cauchy sequence in $X$ by Lemma 4.5 .

Thus the sequence $\left(f\left(x_{1}\right), f\left(y_{1}\right), f\left(x_{2}\right), f\left(y_{2}\right), \cdots\right)$ is left $K$-Cauchy and right $K$-Cauchy sequence in $Y$ from the assumption on the map $f$. Hence $\left(f\left(x_{n}\right)\right) \equiv_{q}\left(f\left(y_{n}\right)\right)$ in $Y$ by Lemma 4.5 .

$(\Leftarrow)$ Assume that $f$ preserves equivalent sequences. Let $\left(x_{n}\right)$ be a left $K$-Cauchy sequence and right $K$-Cauchy in $X$. Since $\left(x_{n}\right) \equiv_{q}\left(x_{n}\right)$, then we have that $\left(f\left(x_{n}\right)\right) \equiv_{q}\left(f\left(x_{n}\right)\right)$ in $Y$. Therefore, the sequence $\left(f\left(x_{n}\right)\right)$ is left $K$-Cauchy and right $K$-Cauchy sequence in $Y$.

Theorem 4.10. Let $(X, q)$ and $(Y, p)$ be quasi-pseudometric spaces and $f:(X, q) \rightarrow(Y, p)$ be a map. Then the following hold.

(1) If the map $f$ is uniformly continuous, then $f$ is left $K$-Cauchy (right K-Cauchy) sequentially-regular.

(2) If the map $f$ is right $K$-Cauchy and right $K$-Cauchy sequentially-regular, then $f$ is continuous with respect to $\tau\left(q^{s}\right)$ and $\tau\left(p^{s}\right)$.

Proof. (1) Let $\epsilon>0$. Suppose that $f$ is uniformly continuous. We only show that $f$ is left $K$-Cauchy sequentially regular and for $f$ right $K$-Cauchy will follow by symmetry. Let $\left(x_{n}\right)$ be any left $q$ - $K$-Cauchy sequence in $X$.

Then there exists $\delta>0$ because $f$ is uniformly continuous such that

$$
q\left(x_{k}, x_{n}\right)<\delta \text { whenever } N \leq k \leq n
$$

for some $N \in \mathbb{N}$ since $\left(x_{n}\right)$ is left $K$-Cauchy sequence in $X$. It follows that

$$
p\left(f\left(x_{k}\right), f\left(x_{n}\right)\right)<\epsilon \text { whenever } N \leq k \leq n
$$

for some $N \in \mathbb{N}$. Hence $\left(f\left(x_{n}\right)\right)$ is left $K$-Cauchy in $Y$.

(2) Suppose that $f$ is right $K$-Cauchy and right- $K$-Cauchy sequentially-regular. If $\left(x_{n}\right)$ be sequence in $X$ such that $\left(x_{n}\right)$ is $q^{s}$-convergent to $a \in X$. We show that the sequence $\left(f\left(x_{n}\right)\right)$ is $p^{s}$-convergent to $f(a)$.

We consider the constant sequence $(a)$ which is $q^{s}$-convergent to $a$. Then we have that $\left(x_{n}\right) \equiv_{q}(a)$ by Lemma 4.2. It follows that the sequence $\left(x_{1}, a, x_{2}, a, \cdots\right)$ is left $K$-Cauchy and right $K$-Cauchy ( $q^{s}$-Cauchy) in $X$.

From our assumption we have $\left(f\left(x_{1}\right), f(a), f\left(x_{2}\right), f(a), \cdots\right)$ is left $K$-Cauchy and right $K$-Cauchy ( $p^{s}$-Cauchy) in $Y$ with a convergent subsequence $(f(a))$ which $p^{s}$-convergent to $f(a)$. Thus the sequence $\left(f\left(x_{n}\right)\right)$ is $p^{s}$-convergent to $f(a)$.

Example 4.11 (compare [15, Example 1]). Let $X=\{0\} \cup\{1 / n: n \in \mathbb{N}\} \cup\{n: n \in \mathbb{N} \backslash\{1\}\}$. We equip $X$ with the quasi-metric $q$ defined by $q(x, x)=0$ for any $x \in X, q(0,1 / n)=1 / n$ for any $n \in \mathbb{N}, q(1 / n, 1 / m)=1 / n$ whenever $n<m, q(0, n)=2^{-n}$ whenever $n \in \mathbb{N} \backslash\{1\}$, $q(n, m)=\left|2^{-1}-2^{-m}\right|$ whenever $n, m \in \mathbb{N} \backslash\{1\}$ and $q(x, y)=1$ otherwise.

It is easy to see that sequences $(1 / n)$ and $(n)$ are left $q$ - $K$-Cauchy in $X$ and both are $q$-convergent to 0 . If we consider the function $g:(X, q) \rightarrow(X, q)$ defined by

$$
g(x)=\left\{\begin{array}{lll}
0 & \text { if } \quad x=0 \\
1 / n & \text { if } \quad x=n \in \mathbb{N} \\
n & \text { if } \quad x=1 / n \text { and } n \in \mathbb{N} \backslash\{1\} .
\end{array}\right.
$$


Then the function $g$ preserves left $q$ - $K$-Cauchy sequences since $g((1 / n))=(n)$ and $g((n))=$ $(1 / n)$ and $g$ is continuous.

Theorem 4.12. Let $(X, q)$ and $(Y, p)$ be quasi-pseudometric spaces and $f:(X, q) \rightarrow(Y, p)$ be a uniformly continuous map. Then $f:(X, q) \rightarrow(Y, p)$ is left $q-K$-Cauchy sequentiallyregular if and only if $f:\left(X, q^{t}\right) \rightarrow\left(Y, p^{t}\right)$ is right $q^{t}-K$-Cauchy sequentially-regular.

Proof. We only prove the necessary condition and the sufficiant condition follows by similar arguments. It is obvious that $f:(X, q) \rightarrow(Y, p)$ is uniformly continuous if and only if $f:\left(X, q^{t}\right) \rightarrow\left(Y, p^{t}\right)$ is uniformly continuous.

Suppose that $f:(X, q) \rightarrow(Y, p)$ is left $q$ - $K$-Cauchy sequentially-regular and let $\left(x_{n}\right)$ be a right $q^{t}-K$-Cauchy sequence. Then the sequence $\left(x_{n}\right)$ is left $q-K$-Cauchy in $X$ by Remark $2.2(1)$.

Moreover, the sequence $\left(f\left(x_{n}\right)\right)$ is left $p$ - $K$-Cauchy in $Y$ from the assumption. But the sequence $\left(f\left(x_{n}\right)\right)$ is right $p^{t}$-K-Cauchy in $Y$ again by Remark $2.2(1)$. Hence $f:\left(X, q^{t}\right) \rightarrow$ $\left(Y, p^{t}\right)$ is right $q^{t}-K$-Cauchy sequentially-regular.

Theorem 4.13. Let $(X, q)$ and $(Y, p)$ be quasi-pseudometric spaces. If the uniformly continuous map $f:(X, q) \rightarrow(Y, p)$ is left $K$-Cauchy and right $K$-Cauchy sequentiallyregular, then $f:\left(X, q^{s}\right) \rightarrow\left(Y, p^{s}\right)$ is $q^{s}$-Cauchy sequentially regular.

Proof. Let $\left(x_{n}\right)$ be a $q^{s}$-Cauchy sequence in $X$. Then $\left(x_{n}\right)$ is both left and right $q-K$ Cauchy in $X$ by Remark $2.2(2)$.

Furthemore, $\left(f\left(x_{n}\right)\right)$ is both left and right $p$ - $K$-Cauchy in $Y$ since $f:(X, q) \rightarrow(Y, p)$ is both left $K$-Cauchy and right $K$-Cauchy sequentially-regular.

Moreover, the sequence $\left(f\left(x_{n}\right)\right)$ is $p^{s}$-Cauchy by Remark $2.2(2)$. Therefore, the uniformly continuous map $f:\left(X, q^{s}\right) \rightarrow\left(Y, p^{s}\right)$ is $q^{s}$-Cauchy in $X$.

Theorem 4.14. Let $(X, q)$ and $(Y, p)$ be quasi-pseudometric spaces and $f:(X, q) \rightarrow(Y, p)$ be a map. Then whenever $(X, q)$ is left Smyth complete and the map $f$ is continuous, then $f$ is left K-Cauchy sequentially-regular.

Proof. Suppose that $(X, q)$ is left Smyth complete and the map $f$ is continuous. If the sequence $\left(x_{n}\right)$ is left $K$-Cauchy, then there exists $x \in X$ such that $\left(x_{n}\right)$ is $q^{s}$-convergent to $x$ by the left Smyth completeness of $(X, q)$.

Then, the sequence $\left(f\left(x_{n}\right)\right)$ is $p^{s}$-convergent to $f(x)$ since the map $f:\left(X, q^{s}\right) \rightarrow\left(Y, p^{s}\right)$ is continous. Hence the sequence $\left(f\left(x_{n}\right)\right)$ is $q^{s}$-Cauchy. Therefore, the sequence $\left(f\left(x_{n}\right)\right)$ is left $K$-Cauchy by Remark 2.2(2).

Corollary 4.15. Let $(X, q)$ and $(Y, p)$ be quasi-pseudometric spaces and $f:(X, q) \rightarrow(Y, p)$ be a map. Then whenever $(X, q)$ is right Smyth complete and the map $f$ is continuous, then $f$ is right $K$-Cauchy sequentially-regular.

Remark 4.16. In Theorem 4.14, if we replace the left Smyth completeness by the sequentially left $K$-completeness, the theorem does not hold because for a sequence being left $K$-Cauchy does not guarantee the existence of the limit (see [13, Example 2]).

\section{Total boundedness and left $K$-Cauchy sequential regularity}

Let $(X, q)$ be a quasi-pseudometric space. An arbitrary subset $A$ of $X$ is called $q$-bounded if and only if there exists $x \in X, r>0$ and $s>0$ such that $A \subseteq D_{q}(x, r) \cap D_{q^{t}}(x, s)$. Note that one can replace $D_{q}(x, r) \cap D_{q^{t}}(x, s)$ by $D_{q}[x, r] \cap D_{q^{t}}[x, s]$. 
Note that the above definition is slightly different from [16]. In the sense of [16] a subset $A$ of $X$ can be $q$-bounded and not necessary $q^{t}$-bounded. Obviously in our context a subset $A$ is $q$-bounded if and only if it is $q^{t}$-bounded. But $q$-boundedness (or $q^{t}$-boundedness) does not imply $q^{s}$-boundedness. Moreover, if $q$ is an extended quasi-pseudometric on $X$ (i.e. the distance between two point can be $\infty$ ), then a subset $B$ of $X$ can be included in $D_{q}(x, \epsilon)$ for some $x \in X$ but its diameter $\operatorname{diam}(B)=\{q(y, z): y, z \in B\}=\infty$ (see [16, p. 2022]).

Let $\mathscr{B}_{q}(X)$ be the collection of all $q$-bounded subsets of $X$ whenever $(X, q)$ is quasipseudometric space. It is easy to see that

(a) whenever $x \in X$, then $\{x\} \in \mathscr{\mathscr { P }}_{q}(X)$,

(b) whenever $A \subseteq B \subseteq X$ and $B \in \mathscr{\mathscr { B }}_{q}(X)$, then $A \in \mathscr{\mathscr { B }}_{q}(X)$,

(c) whenver $A, B \in \mathscr{\mathscr { B }}_{q}(X)$, then $A \cup B \in \mathscr{\mathscr { B }}_{q}(X)$.

It follows that $\mathscr{B}_{q}(X)$ forms a bornology on $X$ and this bornology is called the quasimetric bornology determined by $q$. Furthermore, We have the following observations instead of the one observed in [11]

$$
\mathscr{B}_{q^{s}}(X)=\mathscr{B}_{q}(X)
$$

and

$$
\mathscr{B}_{q^{s}}(X)=\mathscr{B}_{q^{t}}(X) \text {. }
$$

Remark 5.1. Let $(X, q)$ be a quasi-pseudometric space and $A \subseteq X$. It is easy to see that:

(i) If $A \in \mathscr{\mathscr { P O } _ { q }}(X)$, then $A \in \mathscr{B}_{q}(X)$.

(ii) Whenever $F$ is finite subset of $X, F \in \mathscr{T} \mathscr{Q}_{q}(X)$.

Proposition 5.2. Let $(X, q)$ and $(Y, p)$ be quasi-pseudometric spaces and $f:(X, q) \rightarrow$ $(Y, p)$ be a map. Then $\left.f\right|_{T}$ is uniformly continuous, whenever $T \in \mathscr{T O S}_{q}(X)$ if and only if $f$ is Cauchy sequentially regular.

Proof. $(\Rightarrow)$ Assume that $f:(T, q) \rightarrow(Y, p)$ is uniformly continuous with $T$ is $q^{s}$-totally bounded. Let $\left(x_{n}\right)$ be a $q^{s}$-Cauchy sequence. Then $\left\{x_{n}: n \in \mathbb{N}\right\}$ is $q^{s}$-totally bounded and $f:\left(T, q^{s}\right) \rightarrow\left(Y, p^{s}\right)$ is uniformly continuous. It follows that $f$ is Cauchy sequentially regular from [2, Proposition 5.7(2)].

$(\Leftarrow)$ Without loss of generality we suppose that $f:\left(T, q^{s}\right) \rightarrow\left(Y, p^{s}\right)$ is not uniformy continuous and $T$ is $q^{s}$-totally bounded. Then for any $n \in \mathbb{N}$, there exists two sequences $\left(x_{n}\right),\left(t_{n}\right)$ in $T$ such that

$$
q^{s}\left(x_{n}, t_{n}\right)<\frac{1}{n} \text { and } p^{s}\left(f\left(x_{n}\right), f\left(t_{n}\right)\right) \geq \epsilon \text { for some } \epsilon>0 .
$$

From the $q^{s}$-totally boundedness of $T$, suppose that the sequence $\left(t_{n}\right)$ is $q^{s}$-Cauchy, then the sequence $\left(t_{1}, x_{1}, t_{2}, x_{2}, \cdots\right)$ is $q^{s}$-Cauchy but its image $\left(f\left(t_{1}\right), f\left(x_{1}\right), f\left(t_{2}\right), f\left(x_{2}\right), \cdots\right)$ under $f$ is not $p^{s}$-Cauchy from (5.3).

Theorem 5.3. Let $(X, q)$ be a quasi-pseudometric space and $F$ be a nonempty subset of $X$. Then following conditions are equivalent:

(1) $F$ is q-totally bounded;

(2) Whenever $(Y, \| . \mid)$ is an asymmetric normed space and the map $f:(X, q) \rightarrow\left(Y, q_{\| . \mid}\right)$ is left and right $K$-Cauchy sequentially regular, then $f(F) \in \mathscr{B}_{q_{\| . \mid}}(Y)$;

(3) Whenever $(Y, \| . \mid)$ is an asymmetric normed space and the map $f:(X, q) \rightarrow\left(Y, q_{\| . \mid}\right)$ is uniformly locally semi-Lipschitz, then $f(F) \in \mathscr{B}_{q_{\| . \mid}}(Y)$;

(4) Whenever the function $f:(X, q) \rightarrow\left(\mathbb{R}, q_{u}\right)$ is uniformly locally semi-Lipschitz, then $f(F)$ is $q_{u}$-bounded set $\mathbb{R}$. 
Proof. (1) $\Longrightarrow(2)$ Suppose $f:(X, q) \rightarrow\left(Y, q_{\| . \mid}\right)$is left and right $K$-Cauchy sequentially regular and $F$ is $q$-totally bounded. We have that $f:\left(X, q^{s}\right) \rightarrow\left(Y, q_{\|\cdot\|}\right)$ is $q^{s}$ Cauchy sequentially regular by Theorem 4.13 . Since $F$ is $q^{s}$-totally bounded as a $q$-totally bounded. Then $f(F) \in \mathscr{\mathscr { B }}_{q_{\|.\|}}(Y)$ by [1, Theorem 3.2]. Thus from inclusion (5.1) we have $f(F) \in \mathscr{O P}_{q_{\| . \mid}}(Y)$.

$(2) \Longrightarrow(3)$ and $(3) \Longrightarrow(4)$ Follows from Lemma 3.5 and [1, Theorem 3.2].

$(4) \Longrightarrow$ (1) Suppose that $F$ is not $q$-totally bounded. We show that there exists a semi-Lipschitz function $g:\left(D_{q}(x, \delta), q\right) \rightarrow\left(\mathbb{R}, q_{u}\right)$ for any $x \in X$ and some $\delta>0$.

Since $F$ is not $q$-totally bounded, then we have that

$$
F \nsubseteq \bigcup_{k=1}^{n} D_{q^{s}}\left(f_{k}, \epsilon\right) \text {, where } f_{k} \in F \text { whenever } k \in\{1, \cdots, n\}
$$

for some $\epsilon>0$. By induction, we construct a sequence $\left(f_{n}\right)$ in $F$ such that whenever $n \in \mathbb{N}$ we have $f_{n+1} \notin \bigcup_{k=1}^{n} D_{q^{s}}\left(f_{k}, \epsilon\right)$.

It follows that the family $\left\{D_{q^{s}}\left(f_{n}, \frac{\epsilon}{4}\right): n \in \mathbb{N}\right\}$ is uniformly discrete. Furthermore, we have for any $x \in X$, there exists $n^{\prime} \in \mathbb{N}$ such that

$$
\emptyset \neq D_{q^{s}}(x, \epsilon / 4) \cap D_{q^{s}}\left(f_{n^{\prime}}, \epsilon / 4\right) \subseteq D_{q}(x, \epsilon / 4) \cap D_{q}\left(f_{n^{\prime}}, \epsilon / 4\right)
$$

from [2, Proposition 3.8].

Let $g$ be a function defined by

$$
g(x)= \begin{cases}n-\frac{4 n}{\epsilon} q\left(f_{n}, x\right) & \text { if } x \in D_{q}\left(f_{n}, \epsilon / 4\right) \\ 0 & \text { otherwise. }\end{cases}
$$

It is obvious that the function $g$ is unbounded with respect to $u$. We now show that $g$ is a semi-Lipschitz. Consider $x, y \in D_{q}\left(f_{n}, \epsilon / 4\right)$, then

$$
\begin{aligned}
q_{u}(g(x), g(y)) & =(g(x)-g(y))^{+}=\left[\left(n-\frac{4 n}{\epsilon} q\left(f_{n}, x\right)\right)-\left(n-\frac{4 n}{\epsilon} q\left(f_{n}, y\right)\right)\right] \\
& =\frac{4 n}{\epsilon}\left[q\left(f_{n}, y\right)-q\left(f_{n}, x\right)\right] \\
& \leq \frac{4 n}{\epsilon} q(x, y) .
\end{aligned}
$$

Therefore, we have that the function $g$ is semi-Lipschitz with $k=\frac{4 n}{\epsilon}$.

\section{References}

[1] G. Beer and M.I. Garrido, Bornologies and locally Lipschitz functions, Bull. Aust. Math. Soc. 90, 257-263, 2014.

[2] G. Beer and S. Levi, Total boundedness and bornologies, Topology Appl. 156, 12711288, 2009.

[3] G. Beer and S. Levi, Strong uniform continuity, J. Math. Anal. Appl. 350, 568-589, 2009.

[4] S. Cobzas, Completeness in Quasi-Pseudometric SpacesA Survey, Mathematics, 8 (8), 1279, 2020.

[5] S. Cobzas, Functional analysis in asymmetric normed spaces, Frontiers in Mathematics, Springers, Basel, 2013.

[6] G. Di Maio, E. Meccariello and S. Naimpally, Decompositions of UC spaces, Questions Answers Gen. Topology, 22, 13-22, 2004. 
[7] D. Doitchinov, On completeness in quasi-metric spaces, Topology Appl. 30, 127-148, 1988.

[8] T. Jain and S. Kundu, Atsuji completions: Equivalent characterisations, Topology Appl. 154, 28-38, 2007.

[9] H-P.A. Künzi, An introduction to quasi-uniform spaces, Contemp. Math. 486, 239304, 2009.

[10] S.P. Moshokoa, On classes of maps with the extension property to the bicompletion in quasi-pseudo metric spaces, Quaest. Math. 28, 391-400, 2005.

[11] O. Olela Otafudu, W. Toko and D. Mukonda, On bornology of extended quasi-metric spaces, Hacet. J. Math. Stat. 48, 1767-1777, 2019.

[12] R.F. Snipes, Functions that preserve Cauchy sequences, Nieuw Arch. Voor Wiskd. 25, 409-422, 1977.

[13] I.L. Reilly, P.V. Subrahmanyam and M.K. Vamanamurthy, Cauchy sequences in quasi-pseudometric spaces, Monatsh. Math. 93, 127-140, 1982.

[14] S. Romaguera and M. Schellekens, Quasi-metric properties of complexity spaces, Topology Appl. 98, 311-322, 1999.

[15] S. Romaguera and P. Tirado, A characterization of quasi-metric completeness in terms of $\alpha \psi$-contractive mappings having fixed points, Mathematics, 8 (1), 16, 2020.

[16] T. Vroegrijk, Pointwise bornological space, Topology Appl. 156, 2019-2027, 2009. 